\title{
Prevalencia de Borrelia burgdorferi sensu lato en roedores sinantrópicos de dos comunidades rurales de Yucatán, México
}

\author{
Analilia Solís-Henández¹, Roger Iván Rodríguez-Vivas", \\ María Dolores Esteve-Gassent², Sandra Luz Villegas-Pérez ${ }^{1}$ \\ 1 Laboratorio de Parasitología, Facultad de Medicina Veterinaria y Zootecnia, Universidad Autónoma de Yucatán, \\ Yucatán, México \\ 2 Department of Veterinary Pathobiology, College of Veterinary Medicine and Biomedical Sciences, Texas A\&M \\ University, College Station, USA
}

Introducción. La enfermedad de Lyme es una zoonosis multisistémica causada por Borrelia burgdorferi sensu lato. Esta espiroqueta circula en un ciclo enzoótico entre un reservorio vertebrado primario y las garrapatas. Se ha encontrado que varias especies de roedores son eficientes reservorios naturales de B. burgdorferi s.l.

Objetivo. Estimar la prevalencia de B. burgdorferi s.I. en roedores sinantrópicos en dos comunidades rurales de Yucatán, México.

Materiales y métodos. Se capturaron 123 roedores (94 Mus musculus y 29 Rattus rattus) para obtener muestras de tejidos de oreja y vejiga. Para la detección de B. burgdorferi s.I. en las muestras, se amplificaron los genes de la flagelina B (fla B) y las lipoproteínas de membrana externa, ospC y p66, mediante reacción en cadena de la polimerasa, y se secuenciaron los amplicones obtenidos.

Resultados. La frecuencia de infección por $B$. burgdorferi s.I. en roedores fue de 36,5\% para flaB (45/123), de 10,5\% (13/123) para p66 y de 3,2 \% (4/123) para ospC. En $R$. rattus la frecuencia de infección fue de $17,2 \%$ y en $M$. musculus fue de $42,5 \%$. La frecuencia de infección de $B$. burgdorferi s.I. en los tejidos estudiados fue de 11,3\% (14/123) en muestras de tejido de vejiga y de $17,0 \%(21 / 123)$ en las de oreja. No se encontraron diferencias estadísticas $(p>0,05)$ en la frecuencia de infección entre los dos tipos de muestras de tejido utilizadas para el diagnóstico. El gen ospC presentó 98 \% de homología con la especie Borrelia garinii, una de las especies heterogéneas del complejo B. burgdorferi s.I.

Conclusiones. Los roedores presentaron una alta prevalencia de infección con B. burgdorferi s.l.; las especies $M$. musculus y $R$. rattus podrían jugar un papel importante en la continuidad de la presencia de esta bacteria en comunidades rurales de Yucatán, México.

Palabras clave: Borrelia burgdorferi, enfermedad de Lyme, roedores, asentamientos rurales, México. doi: http://dx.doi.org/10.7705/biomedica.v36i3.3139

\section{Prevalence of Borrelia burgdorferi sensu lato in synanthropic rodents in two rural communities of Yucatán, México}

Introduction: Lyme disease is a multisystemic zoonotic disease caused by Borrelia burgdorferi sensu lato. This spirochete circulates in an enzootic cycle between the primary vertebrate reservoir and its tick vectors. Different species of rodents are known to be efficient natural reservoirs for $B$. burgdorferi s.l.

Objective: To estimate the prevalence of $B$. burgdorferi s.l. in synanthropic rodents from two rural communities of Yucatán, México.

Materials and methods: A total of 123 rodents (94 Mus musculus and 29 Rattus rattus) were trapped, and ear and bladder samples were collected. Flagelin $\mathrm{B}(\mathrm{fla} B)$ genes and outer membrane lipoproteins ospC y $p 66$ were amplified in order to detect $B$. burgdorferi s.l. presence in the samples. The obtained amplicons were sequenced.

Results: The overall infection rates in rodents were $36.5 \%$ for flaB (45/123), $10.5 \%(13 / 123)$ for p66, and $3.2 \%$ (4/123) for ospC. Rattus rattus had $17.2 \%$ of infection and M. musculus, $42.5 \%$. From all examined tissue, $11.3 \%(14 / 123)$ of bladders, and $17.0 \%(21 / 123)$ of ears were infected with the

\section{Contribución de los autores:}

Analilia Solís-Hernández, Roger Iván Rodríguez-Vivas y María Dolores Esteve-Gassent: diseño del estudio, análisis e interpretación de los resultados

Sandra Luz Villegas-Pérez: análisis de las muestras mediante pruebas moleculares

Todos los autores participaron en la ejecución de los aspectos prácticos del estudio y en la escritura del manuscrito. 
spirochete Borrelia burgdorferi s.I. No statistical differences ( $p>0.05)$ were found between the two tissue samples used for diagnosis. The ospC gen was $98 \%$ homologous to Borrelia garinii, one species of the B. burgdorferi s.l. complex.

Conclusions: We concluded that rodents have a high prevalence of $B$. burgdorferi s.I. infection, and both species of rodents, $M$. musculus and $R$. rattus, might be playing an important role in the maintenance of this bacterium in rural communities of Yucatán, México.

Key words: Borrelia burgdorferi, Lyme disease, rodentia, rural settlements, México. doi: http://dx.doi.org/10.7705/biomedica.v36i3.3139

La enfermedad de Lyme es causada por varias especies del complejo Borrelia burgdorferi sensu lato, incluida $B$. garinii, y es transmitida por garrapatas del género Ixodes. La enfermedad ha cobrado especial importancia a nivel mundial por su alta incidencia, su extensa distribución y la gravedad de sus manifestaciones clínicas.

Hasta el momento, los Centers for Disease Control and Prevention (CDC) de Estados Unidos solo reconocen la presencia de la enfermedad de Lyme en Estados Unidos y en Eurasia, pero no en Centroamérica y Suramérica (1). La importancia de la enfermedad de Lyme en México no se ha esclarecido a pesar de los distintos estudios que se han hecho sobre la distribución de la garrapata Ixodes, no solo en los estados del noreste mexicano, incluida la zona fronteriza de Texas y México (2), sino a lo largo del centro y sureste del país y en la península de Yucatán $(3,4)$ (GordilloPérez G, Vargas M, Sosa C, Lima-Eder S, MineroGonzález E, Lara B, et al. Prevalencia de infección de $B$. burgdorferi y Ehrlichia spp. en garrapatas y roedores provenientes de tres parques nacionales del centro de la República Mexicana. Primer Congreso Latinoamericano de Acarología. 20 al 23 de mayo de 2012, Puebla, México). La presencia de la bacteria también se ha documentado en huéspedes como el venado cola blanca, en roedores silvestres de tres parques nacionales del centro de México y en perros de Baja California $(5,6)$ (Gordillo-Pérez G, Vargas M, Sosa C, Lima-Eder $\mathrm{S}$, Minero-González E, Lara B, et al. Prevalencia de infección de $B$. burgdorferi y Ehrlichia spp. en garrapatas y roedores provenientes de tres parques nacionales del centro de la República Mexicana. Primer Congreso Latinoamericano de

\footnotetext{
Correspondencia:

Roger Iván Rodríguez-Vivas, Facultad de Medicina Veterinaria y Zootecnia, Km 15.5 carretera Mérida-Xmatkuil, CP 97000, Mérida, Yucatán, México

Teléfono: (52-9999) 423200

rvivas@correo.uady.mx

Recibido: 17/12/15; aceptado: 17/03/16
}

Acarología. 20 al 23 de mayo de 2012, Puebla, México). Recientemente, Solís, et al. (7), reportaron que Ixodes affinis se encuentra como parásito en perros de comunidades rurales de Yucatán, México, y la mayor infestación se presenta en los que son llevados a las áreas selváticas cuando salen de cacería con sus dueños. Asimismo, Rodríguez, et al. (8), encontraron que I. affinis parasita animales domésticos (perros, gatos, caballos) y animales silvestres (ocelote, zopilote) en Yucatán. En Estados Unidos se ha reportado que I. scapularis e I. pacificus son los vectores responsables de la transmisión de B. burgdorferi s.l. en humanos y en animales silvestres y de compañía, mientras que $I$. affinis está principalmente involucrada en la transmisión de este patógeno entre animales de vida silvestre (9).

Estos estudios sobre la distribución y la amplia gama de huéspedes de I. affinis, así como los casos de la enfermedad de Lyme confirmados en humanos y la seroprevalencia nacional de 1,1\%, demuestran la existencia de un ciclo zoonótico responsable de esta enfermedad en México $(10,11)$.

El mantenimiento de $B$. burgdorferis.l., así como de otros agentes patógenos zoonóticos transmitidos por garrapatas, depende de la abundancia de los reservorios y vectores competentes, y de la interacción entre huésped y vector, por lo cual la relación entre garrapatas del género Ixodes, la espiroqueta y los mamíferos huésped ha sido objeto de numerosos estudios $(12,13)$. En Europa y Estados Unidos se han detectado muchas especies de mamíferos como reservorios competentes, pero a los roedores se los ha implicado como los principales reservorios naturales de $B$. burgdorferi s.l. y $B$. burgdorferi sensu stricto, ya que algunas especies pueden mantener la bacteria durante periodos prolongados (14-16).

Las comunidades de ectoparásitos y patógenos de diferentes especies de roedores de vida silvestre se han estudiado extensamente $(17,18)$, pero muy poco se conoce sobre las especies de roedores sinantrópicos. Los roedores sinantrópicos, como el 
ratón de casa (Mus musculus) y la rata negra (Rattus rattus), son especies asociadas a las viviendas y a sistemas agrícolas y pecuarios. Mihalca, et al. (19), mencionan que en Rumania la infestación con garrapatas en estos roedores es baja, y que tienen poca importancia como reservorios de patógenos. Sin embargo, la asociación de pequeños mamíferos y garrapatas se ha estudiado poco en esta región, a pesar de ser el único país de Europa con cinco biorregiones y una amplia biodiversidad (especialmente de roedores). Por otra parte, Bhide, et al. (20), y Richter, et al. (21), mencionan que los roedores sinantrópicos son fuente de alimento para estadios inmaduros y pueden ser reservorios de agentes como Borrelia spp. en un entorno urbano o rural, y que tienen un ciclo doméstico en el cual los humanos y los animales pueden ser huéspedes accidentales.

En México se ha detectado $B$. burgdorferi s.I. en roedores silvestres como Peromyscus spp. $(5,11)$; sin embargo, se desconoce si los roedores sinantrópicos $R$. rattus y $M$. musculus son reservorios de esta bacteria. Por tal motivo, el objetivo del presente estudio fue estimar la prevalencia de $B$. burgdorferi s.I. en tejidos de vejiga y oreja de roedores capturados en dos áreas rurales del estado de Yucatán, México.

\section{Materiales y métodos}

\section{Área de estudio}

El estudio se llevó a cabo en las comunidades rurales de Opichen y Tixmehuac en el estado de Yucatán, México.

Opichen tiene una superficie de $268,2 \mathrm{~km}^{2}$ y se localiza entre los paralelos $20^{\circ} 26^{\prime \prime}$ y $20^{\circ} 36^{\prime \prime}$ de latitud norte y los meridianos $89^{\circ} 22^{\prime \prime}$ y $89^{\circ} 46^{\prime \prime}$ de longitud oeste. El clima es cálido subhúmedo con lluvias en verano, presenta una temperatura media anual de $28{ }^{\circ} \mathrm{C}$ y una precipitación pluvial anual de $1.100 \mathrm{~mm}$, y predominan los vientos provenientes del sureste. La comunidad está rodeada de parches de selva baja caducifolia, milpas y pastizales.

Tixmehuac tiene una superficie de $251,6 \mathrm{~km}^{2}$, y se localiza entre los paralelos $20^{\circ} 08^{\prime \prime}$ y $20^{\circ} 19^{\prime \prime}$ de latitud norte y los meridianos $89^{\circ} 05^{\prime \prime}$ y $89^{\circ} 12^{\prime \prime}$ de longitud oeste. El clima es cálido subhúmedo con lluvias en verano. La temperatura media anual es de $26{ }^{\circ} \mathrm{C}$, la precipitación pluvial anual es de 1.050 $\mathrm{mm}$ y los vientos dominantes soplan en dirección sureste-noroeste. La vegetación prevaleciente en la periferia es selva mediana subcaducifolia (22).

\section{Selección de viviendas y captura de roedores}

En cada comunidad se seleccionaron por conveniencia 50 viviendas, las cuales se dividieron en cuatro cuadrantes, tomando como referencia las calles principales que cruzan los poblados de norte a sur y de este a oeste. Cada vivienda seleccionada se ubicó por georreferencia y se solicitó la anuencia de los dueños para participar en el estudio. Cuando algún propietario no aceptó participar, se seleccionó otra vivienda. Los muestreos se hicieron entre agosto y noviembre del 2013.

Para la captura de los roedores se emplearon trampas Sherman $(7,5 \times 23 \times 9 \mathrm{~cm}$, HB Sherman Traps Inc., Tallahassee, Florida, USA). En cada vivienda se colocaron seis trampas distribuidas en el peridomicilio y el domicilio, las cuales se dejaron abiertas durante una noche para lograr un esfuerzo de captura de 1.200 noches por trampa y por localidad. Como cebo se utilizó una mezcla de hojuelas de avena y esencia de vainilla. Las trampas se inspeccionaron en la mañana del siguiente día y los animales capturados fueron trasladados al Laboratorio de Parasitología del Campus de Ciencias Biológicas y Agropecuarias de la Universidad Autónoma de Yucatán. La captura y el manejo de los roedores se ajustaron a los lineamientos para el uso de animales silvestres de la investigación de la American Society of Mammalogists (23).

Se registraron las medidas somáticas convencionales (peso, largo total, largo de cola, pata y oreja) de cada roedor capturado; para determinar la especie se empleó la guía para mamíferos de Centroamérica y sureste de México (24). Los roedores capturados fueron anestesiados por vía intraperitoneal con pentobarbital sódico $(65 \mathrm{mg} / \mathrm{kg})$. A todos se les revisó a contra pelo en búsqueda de ectoparásitos.

A continuación, se aplicó la eutanasia por dislocación cervical en roedores pequeños o por sobredosis anestésica en los roedores más grandes. Después de la eutanasia, se abrió la cavidad abdominal asépticamente y se extrajo la vejiga urinaria (de aproximadamente 3 por 3 por $0,2 \mathrm{~mm}$ de grosor) y una muestra de la oreja (de aproximadamente 2 por 2 por $0,4 \mathrm{~mm}$ de grosor). Las muestras se mantuvieron congeladas a $-80^{\circ} \mathrm{C}$ hasta su procesamiento, con la metodología descrita por Goodman, et al. (25). Para evitar la contaminación entre muestras, la superficie de los instrumentos de laboratorio se limpió con una solución libre de ADNasas (DNAZap ${ }^{\mathrm{TM}}$, Termo Fisher Scientific Inc., Waltham, USA). 


\section{Extracción de ADN}

Se extrajo el ADN de todas las muestras de tejido de vejiga y oreja usando el kit comercial DNeasy Blood and Tissue (Qiagen, Valencia, USA) y siguiendo las instrucciones del fabricante. Posteriormente, las muestras se almacenaron a $-80^{\circ} \mathrm{C}$ hasta su uso.

\section{Diagnóstico molecular}

Se utilizó un termociclador Minicycler (BIO RAD) para todas las pruebas de reacción en cadena de la polimerasa (PCR) convencional y anidada. Para detectar la presencia del género Borrelia en las muestras de oreja y vejiga de los roedores, se utilizó como marcador genético la flagelina cromosómica de 41-kDa (flaB, bb0141) en una PCR convencional según la metodología descrita por Jaulhac (26), usando los iniciadores F5'AACACACCAGCATCACTTTCAGG3' y R5'GAG AATTAACTCCGCCTTGAGAAGG3'. Los productos positivos para flaB se amplificaron con el fin de identificar $B$. burgdorferi s.l. utilizando los genes p66 (66-kDA) y ospC (23-kDA) en PCR anidadas con la metodología descrita por Bunikis, et al. $(27,28)$. Para la amplificación del gen $p 66$ se utilizaron los siguientes iniciadores: F5 'GATTTTTCTATATTTGGACACAT 3', R5'TGTAAATCTTATTAGTTTTTCAAG3', Fn5'CAA AAAAGAAACACCCTCAGATCC3' y Rn5'CCT GTTTTTAAATAAATTTTTGTAGCATC3', y para ospC: F5'ATGAAAAAGAATACATTAAGTG C3', R 5'ATTAATCTTATAATATTGATTTTAATTAAGG3', Fn5'TATTAATGACTTTATTTTTATTTATATCT3' y Rn5'TTGATTTTAATTAAGGTTTT TTTGG3'.

El tamaño de los productos esperados para cada uno de los genes flaB, p66 y ospC fue de 235 pb, $684 \mathrm{pb}$ y 585 pb, respectivamente. Como control positivo se usó el ADN genómico de $B$. burgdorferi sensu stricto B31 (cepas MSK5 y A3), $B$. afzelii (cepa 51567), B. garinii (cepa 51383) y B. turicatae (cepas TCBP2 y 91E135), proporcionado por la Universidad de Texas A\&M en College Station, Texas (EUA); para evitar una posible contaminación, en cada reacción se incluyó un control negativo. Para cada producto de PCR se hizo electroforesis en gel de agarosa al $2 \%$, que se tiñó con bromuro de etidio y se examinó mediante transiluminación ultravioleta.

\section{Secuenciación}

Los productos de PCR positivos para los genes flaB, p66 y ospC (cuatro muestras) se purificaron usando el kit comercial DNeasy Blood and Tissue, protocolo para purificación (Qiagen, Valencia, USA) y siguiendo las instrucciones del fabricante. Posteriormente, las muestras fueron enviadas al Centro de Investigación y Desarrollo en Ciencias de la Salud (NOVOGEN) en Monterrey, Nuevo León, México, para su secuenciación. La búsqueda de secuencias homólogas de B. burgdorferi s.I. se hizo en el GenBank mediante el programa Chromas 223. Los aminoácidos se tradujeron a partir de la secuencia de nucleótidos.

\section{Análisis estadístico}

La prevalencia de roedores positivos para $B$. burgdorferi s.l. se obtuvo usando los marcadores genéticos flaB, p66 y ospC. Para conocer la diferencia en la frecuencia de ratas y ratones positivos para B. burgdorferi s.I. en ambas comunidades (Opichen y Tixmehuac) y en los tejidos de vejiga y oreja de las especies de ratas y ratones, se usó la prueba de ji al cuadrado.

\section{Resultados}

En las 100 viviendas estudiadas se capturaron 123 roedores y el mayor número de capturas se obtuvo en la comunidad de Opichen; 94 especímenes correspondían a la especie $M$. musculus, la cual fue la más abundante, y 29 , a la especie $R$. rattus (cuadro 1).

El $36,5 \%(45 / 123)$ de los roedores estudiados fueron positivos para el gen flaB y, de ellos, $41,3 \%$ (19/46) se capturó en la comunidad de Tixmehuac y $33,7 \%(26 / 77)$ en la comunidad de Opichen. No hubo diferencias significativas $(p>0,05)$ entre los casos positivos en las dos comunidades. La frecuencia de infección en $R$. rattus fue de 17,2\% $y$, en $M$. musculus fue de $42,5 \%$ (cuadro 2).

Como se muestra en el cuadro 2, 11,3\% (14/123) de las muestras de vejiga y $17,0 \%(21 / 123)$ de las de oreja presentaban infección con la espiroqueta, en tanto que $12,3 \%$ (10/123) de las muestras fueron positivas en ambos tejidos. No se encontraron

Cuadro 1. Cantidad de individuos de Rattus rattus y Mus musculus capturados en las localidades de Opichen y Tixmehuac en Yucatán, México, para conocer la prevalencia de $B$. burgdorferi sensu lato en roedores sinantrópicos

\begin{tabular}{lccr}
\hline \multirow{2}{*}{ Especie de roedor } & \multicolumn{2}{c}{ Localidad } & \multirow{2}{*}{ Total } \\
\cline { 2 - 3 } & Opichen & Tixmehuac & \\
\hline Mus musculus & 63 & 31 & 94 \\
Rattus rattus & 14 & 15 & 29 \\
Total & 77 & 46 & 123 \\
\hline
\end{tabular}


Cuadro 2. Resultados positivos para el gen flaB obtenidos mediante PCR de tejido de vejiga y oreja de los especímenes de Rattus rattus y Mus musculus capturados en las localidades rurales de Opichen y Tixmehuac, Yucatán, México

\begin{tabular}{lcccccc}
\hline $\begin{array}{l}\text { Especies de } \\
\text { roedores }\end{array}$ & $\begin{array}{c}\text { Total de roedores } \\
\text { estudiados }\end{array}$ & $\begin{array}{c}\text { Total de roedores } \\
\text { positivos }\end{array}$ & $\begin{array}{c}\text { Prevalencia de Borrelia } \\
\text { burgdorferi sensu lato (\%) }\end{array}$ & \multicolumn{2}{c}{ PCR tejido positivo (gen flaB) } \\
\cline { 5 - 8 } & & 40 & 42,5 & 9 & 21 & 10 \\
Mus musculus & 94 & 5 & 17,2 & 4 & 1 & 0 \\
Rattus rattus & 29 & 45 & 36,5 & 13 & 22 & 10 \\
Total & 123 & & & Oreja & Vejiga y oreja \\
\hline
\end{tabular}

diferencias significativas $(p>0,05)$ entre los casos positivos para $B$. burgdorferi spp. en las muestras de ninguno de los dos tipos de tejido.

Entre los roedores positivos para flaB, solo los de la especie $M$. musculus resultaron positivos para el gen $p 66(28,8 \% ; 13 / 45)$ y para el gen ospC (8,8 \%; 4/45); solo $4,4 \%$ de los ratones M. musculus fue positivo para los genes flaB, p66 (figura 1) y ospC (figura 2). El gen ospC tuvo $98 \%$ de homología con la especie $B$. garinii, una de las especies heterogéneas del complejo $B$. burgdorferi s.l. (figura 3).

\section{Discusión}

En las últimas décadas los roedores sinantrópicos han adquirido mayor importancia en la transmisión de diversas enfermedades zoonóticas (29). Su importancia epidemiológica radica en que las poblaciones de estas especies pueden alcanzar gran cantidad de individuos, los cuales son huéspedes de ectoparásitos y sirven como reservorios de agentes patógenos, lo que podría aumentar el riesgo de exposición de los humanos y los animales domésticos (30).

A pesar del estrecho contacto con los humanos, el papel de los roedores sinantrópicos en la epidemiología de Borrelia spp. no ha sido muy estudiado. En México se sabe poco sobre la presencia de esta espiroqueta en roedores y los estudios hechos en el país se han enfocado principalmente en especies silvestres, por lo que el presente trabajo es el primer reporte de la detección de $B$. burgdorferi s.I. en roedores sinantrópicos (M. musculus y $R$. rattus) en Yucatán, México, cuya prevalencia estimada fue de $36,5 \%$ para $B$. burgdorferi s.I., similar a la reportada en China (41 $\%)$ para estas especies (31). Ambas especies de roedores, $R$. rattus y $M$. musculus, se capturaron en el domicilio y peridomicilio y, dada la presencia de vectores competentes, podrían estar involucradas en un posible ciclo doméstico de esta espiroqueta y representar un riesgo de transmisión de Borrelia spp. para los humanos y los animales domésticos.

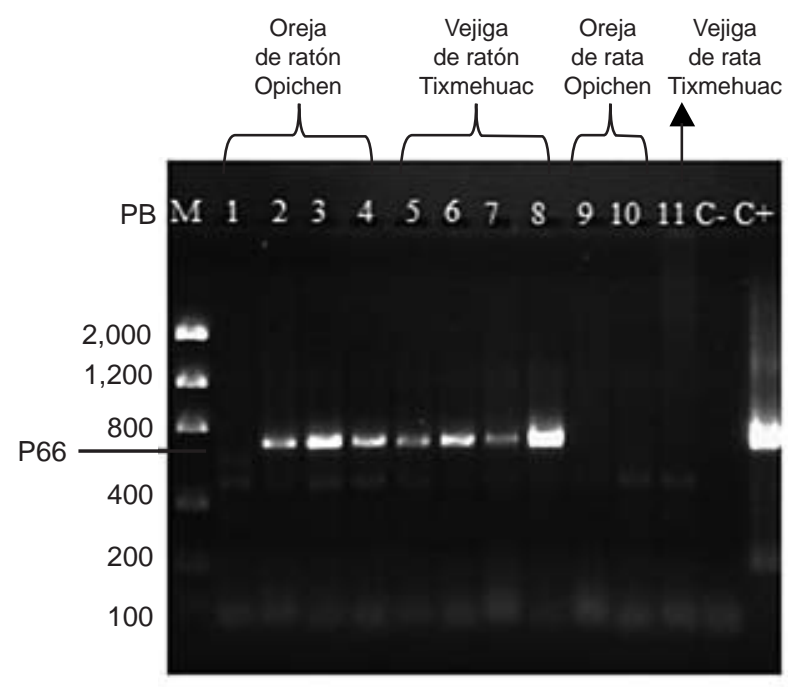

Figura 1. Electroforesis para la amplificación del gen p66 (684 pb) de Borrelia burgdorferi sensu lato en muestras de tejido de oreja y vejiga de individuos de Rattus rattus y de Mus musculus capturados en las localidades rurales de Opichen y Tixmehuac en Yucatán, México. PB: pares de bases; M: marcador de peso molecular; carriles 1, 2, 3 y 4: muestras de oreja de ratón de Opichen (carril 1, muestra negativa, carriles 2, 3 y 4, muestras positivas); carriles $5,6,7$ y 8 : muestras positivas de tejido de vejiga de ratón de Tixmehuac; carriles 9 y 10: muestras negativas de tejido de oreja de rata de Opichen; carril 11: muestra negativa de tejido de vejiga de rata de Tixmehuac; últimos dos carriles: control negativo $(\mathrm{C}-)$ y positivo $(\mathrm{C}+)$ para ADN de Borrelia burgdorferi sensu stricto

Las comunidades seleccionadas para este estudio se caracterizan por presentar dos ambientes diferentes: una vegetación más conservada (selva mediana subcaducifolia) en la comunidad de Tixmehuac que en la de Opichen, en donde hay parches de selva baja caducifolia con tierras cultivadas y pastizales para la ganadería. La fragmentación de la vegetación en esta última comunidad podría relacionarse directamente con la abundancia de ratones, la cual fue mayor (63 M. musculus) que en Tixmehuac (31 M. musculus) (cuadro 1).

En este sentido, Allan, et al. (32), señalan que la creciente superposición del hábitat de los humanos ha traído como consecuencia el aumento de la 
población de roedores, los cuales juegan un papel importante como reservorios de Borrelia spp. Los autores plantearon una hipótesis según la cual los fragmentos pequeños ( $<2$ ha) de bosque 0 selva, comparados con los fragmentos mayores (2 a 8 ha), reducen la diversidad de especies de mamíferos y elevan la densidad de ratones y garrapatas inmaduras, siendo estas últimas un factor de riesgo primario, ya que al alimentarse de dichos reservorios aumentan la probabilidad de adquirir y transmitir la bacteria.

En los 123 roedores capturados en ambas comunidades se recolectaron tres ninfas del género Argasidae. Esta baja infestación puede deberse a

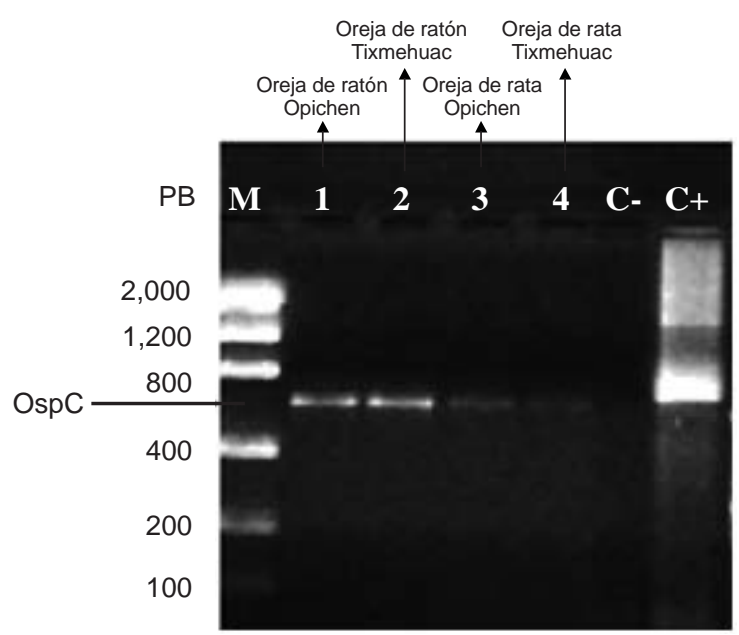

Figura 2. Electroforesis para la amplificación del gen ospC $(585 \mathrm{pb})$ de Borrelia burgdorferi sensu lato en tejido de oreja de individuos de Rattus rattus y Mus musculus capturados en las localidades rurales de Opichen y Tixmehuac en Yucatán, México. PB: pares de bases, M: marcador de peso molecular, carril 1: muestra positiva de tejido de oreja de ratón de Opichen, carril 2: muestra positiva de tejido de oreja de ratón de Tixmehuac, carril 3: muestra negativa de tejido de oreja de rata de Opichen, carril 4: muestra negativa de tejido de oreja de rata de Tixmehuac, últimos dos carriles: control negativo (C-) y positivo (+) para ADN de Borrelia garinii

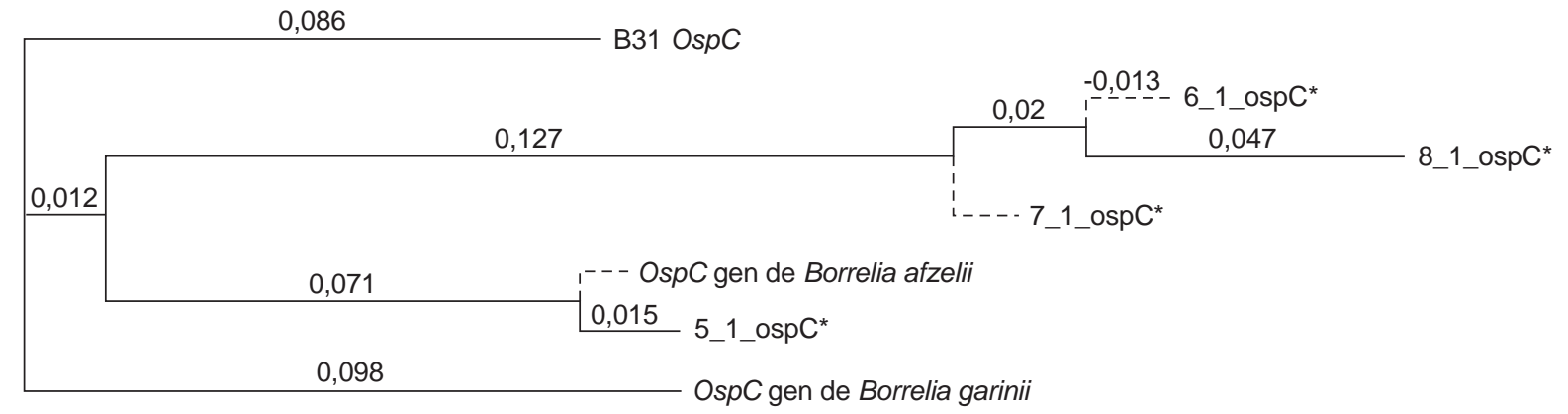

Figura 3. Análisis filogenético bayesiano del gen ospC de Borrelia burgdorferi sensu lato en cuatro muestras positivas para los genes flaB, OspC y p66 (6_1_ospC* , 8_1_ospC* ${ }^{*} 7 \_1 \_o s p C^{*}$ y 5_1_ospC*) que las garrapatas tienen un acentuado carácter estacional y dependen, entre otros factores, de la temperatura ambiental, por lo que es a principios de la primavera cuando tiene lugar la alimentación de los ejemplares que han sobrevivido al invierno (principalmente adultos); la infestación alcanza su máximo nivel durante el verano, tras la eclosión de los huevos y la aparición de una nueva generación de individuos inmaduros, situación que disminuye progresivamente durante el otoño (33).

Esto coincide con los muestreos del presente estudio, los cuales se hicieron a finales del verano e inicio del otoño. Por otro lado, solo se inspeccionaron los individuos capturados y en ellos no se recolectaron de manera cuidadosa los ectoparásitos. Los datos previamente publicados por Mihalca, et al. (19), indican que los roedores sinantrópicos rara vez son parasitados por garrapatas, lo que pone en duda su papel como reservorios competentes de Borrelia spp. Sin embargo, en otros estudios se ha encontrado que los roedores sinantrópicos pueden albergar agentes patógenos zoonóticos como B. burgdorferi, ya que una ninfa infectada sería suficiente para perpetuar la presencia de la bacteria en un entorno urbano o rural $(21,30)$. Además de la transmisión vectorial, en los estudios de Anderson, et al. (34), y Burgess, et al. (35), se comprobó mediante el cultivo de $B$. burgdorferi y de análisis moleculares que, en ausencia de garrapatas, la transmisión transplacentaria puede ser otra vía para el mantenimiento de Borrelia spp. en poblaciones de roedores como $M$. musculus y Peromyscus leucopus, lo cual puede explicar la alta prevalencia y la baja infestación con garrapatas.

En el presente estudio no se encontraron diferencias significativas en la prevalencia de $B$. burgdorferi $\mathrm{s}$.l. en las muestras de tejido de vejiga $(11,3 \%)$ y de oreja $(17,0 \%)$ de los roedores, resultados que 
coinciden con los hallazgos de Petney, et al. (16), en Estados Unidos, en donde dicha prevalencia fue similar. En Europa, en cambio, el tejido de vejiga resultó ser más sensible en la detección del agente patógeno que las muestras de tejido de oreja, lo que sugiere que hay diferencias significativas entre estos tejidos en cuanto a la densidad de bacterias $(36,37)$. La prevalencia estimada de $B$. burgdorferi s.l. en roedores difiere según el método de biopsia y la especie de roedor. La precisión de los diversos procedimientos analíticos utilizados para acumular datos epidemiológicos, debe garantizarse para cada tipo de tejido, para cada especie y para cada genoespecie de $B$. burgdorferi s.l., con el fin de evitar sesgos difíciles de detectar y eliminar posteriormente $(15,38)$.

Con base en los resultados de la secuenciación (figura 3), el gen ospC presentó una similitud y homología de $98 \%$ con la especie $B$. garinii, considerada endémica de Eurasia, la cual es causante de la enfermedad de Lyme con $B$. afzelii y $B$. burgdorferi sensu stricto (39). Rudenko, et al. (40), revelaron en su estudio que los alelos de esta lipoproteína (ospC), que solo se conocían en Europa, se encontraban ampliamente distribuidos en el sureste de Estados Unidos, lo cual respaldaría la hipótesis sobre una posible migración de especies de Borrelia entre continentes (41), probablemente, a través de la ruta de aves migratorias como las paseriformes neotropicales y las aves marinas, que tienen un importante papel como dispersoras de garrapatas infectadas y han generado nuevos focos enzoóticos (42-44). Un ejemplo es la especie $B$. garinii, la cual se ha detectado en garrapatas capturadas en colonias de aves marinas en las costas de Norteamérica (39). Aunado a esto, Steere, et al. (45), han señalado que el aumento en el número de casos de la enfermedad de Lyme a nivel mundial puede atribuirse a la expansión o a la transferencia de genes, lo que ha dado lugar a genotipos recombinantes. Este sería el caso de la genoespecie identificada en Yucatán, B. garinii, conocida por ser la más heterogénea de todas las especies del complejo $B$. burgdorferi s.I. $(46,47)$.

La circulación de $B$. burgdorferis.I. en los llamados focos silenciosos demuestra la complejidad de su ecología y la variedad de los nichos ecológicos que puede ocupar, sin contar con los focos enzoóticos que aún faltan por descubrir (48). Los futuros estudios deberían concentrarse en el papel de los roedores en la transmisión de $B$. burgdorferi s.l. a los humanos y de las garrapatas en su transmisión vectorial.
En conclusión, los roedores sinantrópicos presentaron una alta prevalencia de infección con $B$. burgdorferi s.l. y ambas especies de roedores, $M$. musculus y $R$. rattus, podrían jugar un papel importante en el mantenimiento de esta bacteria en comunidades rurales de Yucatán, México.

\section{Agradecimientos}

Al Consejo Nacional de Ciencia y Tecnología (CONACYT) por proporcionar una beca de doctorado a Analilia Solís-Hernández. Agradecemos de forma especial a las autoridades y habitantes de las comunidades de Opichen y Tixmehuac por permitirnos trabajar en sus casas durante el estudio.

\section{Conflicto de intereses}

Los autores declaran que durante este trabajo no tuvieron conflicto de intereses alguno.

\section{Financiación}

Esta investigación fue financiada por la Universidad Autónoma de Yucatán con recursos del Laboratorio de Parasitología Veterinaria.

\section{Referencias}

1. Centers for Disease Control and Prevention. Lyme disease - 2015. Fecha de consulta: 22 de enero de 2015. Disponible en: www.cdc.gov/lyme.

2. Feria TP, Castro I, Gordillo G, Cavazos AL, Vargas M, Grover A, et al. Implications of climate change on the distribution of the tick vector Ixodes scapularis and risk for Lyme disease in the Texas-Mexico transboundary region. Parasit Vect. 2014;7:199. http://dx.doi.org/10.1186/17563305-7-199

3. Guzmán C, Robbins RG, Pérez TM. The Ixodes (Acari: Ixodidae) of Mexico: Parasite-host and host-parasite checklists. Zootaxa. 2007;1553:47-58.

4. Illoldi P, Rivaldi CL, Sissel B, Trout-Fryxell R, GordilloPérez G, Rodríguez-Moreno A, et al. Species distribution models and ecological suitability analysis for potential tick vectors of Lyme disease in México. J Trop Med. 2012;2012:10. http://dx.doi.org/10.1155/2012/959101

5. Martínez A, Salinas A, Martínez F, Cantu A, Miller DK. Serosurvey for selected disease agents in white-tailed deer from México. J Wildl Dis. 1999;35:799-803. http://dx.doi. org/10.7589/0090-3558-35.4.799

6. Tinoco L, Quiroz H, Quintero MT, Rentería-Evangelista TM, Barreras-Serrano A, Hori-Oshima, S, et al. Prevalence and risk factors for Borrelia burgdorferi infection in Mexicali, Baja California, a México-US border city. Intern J Appl Res Vet Med. 2008;6:161-5.

7. Solís A, Rodríguez RI, Barrera MA, Esteve MD, Apanaskevich DA. Ixodes affinis (Acari: Ixodidae) in dogs from rural localities of Yucatán, México: Prevalence, abundance and associated factors. Vet Mex.2015;2:3. 
8. Rodríguez-Vivas RI, Apanaskevich DA, Ojeda-Chi MM, Trinidad-MartínezI, Reyes-NoveloE, Esteve-Gassent MD, et al. Ticks collected from humans, domestic animals, and wildlife in Yucatán, México. Vet Parasitol. 2016.215;103-7. http://dx.doi.org/10.1016/j.vetpar.2015.11.010

9. Harrison BA, Rayburn WH Jr, Toliver M, Powell EE, Engber BR, Durden LA, et al. Recent discovery of widespread Ixodes affinis (Acari: Ixodidae) distribution in North Carolina with implications for Lyme disease studies. J Vector Ecol. 2010;35:174-9. http://dx.doi.org/10.1111/j.19487134.2010.00044.x

10. Gordillo G, Torres J, Solórzano F, de Martino S, Lipsker D, Velázquez E, et al. Borrelia burgdorferi infection and cutaneous Lyme disease, México. Emerg Infect Dis. 2007; 13:1556-8. http://dx.doi.org/10.3201/eid1310.060630

11. Gordillo G, Solórzano F. Enfermedad de Lyme. Experiencia en niños mexicanos. Bol Med Hosp Infant Mex. 2010; 67:164-76.

12. Lane RS, Brown RN. Wood rats and kangaroo rats: Potential reservoirs of the Lyme disease spirochete in California. $J$ Med Entomol. 1991;28:299-302.

13. Minnelli A, Bertolotti L, Gern L, Gray J. Ecology of Borrelia burgdorferi sensu lato in Europe: Transmission dynamics in multi-host systems, influence of molecular processes and effects of climate change. FEMS Microbiol Vet. 2012;36:83761. http://dx.doi.org/10.1111/j.1574-6976.2011.00312.x.

14. Gern L, Siegenthaler M, Hu CM, Leuba-García S, Humair PF, Moret J. Borrelia burgdorferi in rodents (Apodemus flavicollis and $A$. sylvaticus): Duration and enhancement of infectivity for Ixodes ricinus ticks. Eur J Epidemiol. 1994;10:75-80.

15. Petney TN, Hassler D, Brückner M, Mailwad $\mathbf{M}$. Comparison of urinary bladder and ear biopsy samples for determining prevalence of Borrelia burgdorferi in rodents in central Europe. J Clin Microbiol. 1996;34:1310-2.

16. Levi T, Kilpatrick AM, Mangel M, Wilmers C. Deer, predators, and the emergence of Lyme disease. Proc Natl Acad Sci USA. 2012;109:10942-7. http://dx.doi.org/10.1073/ pnas. 1204536109

17. Stevenson HL, Bai Y, Kosoy MY, Montenieri JA, Lowell JL, Chu MC, et al. Detection of novel Bartonella strains and Yersinia pestis in prairie dogs and their fleas (Siphonaptera: Ceratophyllidae and Pulicidae) using multiplex polymerase chain reaction. J Med Entomol. 2003;40:329-37. http://dx. doi.org/10.1603/0022-2585-40.3.329

18. Durden LA, Polur RN, Nims T, Banks CW, Oliver JH Jr. Ectoparasites and other epifaunistic arthropods of sympatric cotton mice and golden mice: Comparisons and implications for vector-borne zoonotic diseases. J Parasitol. 2004;90:1293-7. http://dx.doi.org/10.1645/GE-333R

19. Mihalca AD, Dumitrache MO, Sándor AD, Magdaş $C$, Oltean M, Györke A, et al. Tick parasites of rodents in Romania: Host preferences, community structure and geographical distribution. Parasit Vectors. 2012;5:266. http://dx.doi.org/10.1186/1756-3305-5-266

20. Bhide M, Travnicek M, Curlik J, Stefancikova A. The importance of dogs in eco-epidemiology of Lyme borreliosis: A review. Vet Med. 2004;4:135-42.
21. Richter D, Schlee DB, Matuschka FR. Reservoir competence of various rodents for the Lyme disease spirochete Borrelia spielmanii. Appl Environ Microbiol. 2011;77:3565-70. http://dx.doi.org/10.1128/AEM.00022-11

22. Instituto Nacional de Estadística y Geografía. Censo General de Población y Vivienda, 2010-2015. Fecha de consulta: 3 de marzo de 2015. Disponible en: http://www. inegi.org.mx/est/contenidos/proyectos/ccpv/

23. Sikes RS, Gannon WL, Animal Care and Use Committee of the American Society of Mammalogists. Guidelines of the American Society of Mammalogists for the use of wild mammals in research. J Mammal. 2011;92:235-53.

24. Reid FA, FieldA. Guide to the Mammals of Central America \& Southeast México. New York: Oxford University Press; 2009.

25. Goodman J, Jurkovich P, Kodner C, Johnson RC. Persistent cardiac and urinary tract infections with Borrelia burgdorferi in experimentally infected Syrian hamsters. J Clin Microbiol. 1991;29:894-6.

26. Jaulhac B, Heller R, Limbach FX, Hansmann Y, Lipsker D, Monteil $\mathbf{H}$, et al. Direct molecular typing of Borrelia burgdorferi sensu lato species in synovial samples from patients with Lyme arthritis. J Clin Microbiol. 2000;38: 1895-900.

27. Bunikis J, Tsao J, Luke CJ, Luna MG, Fish D, Barbour AG. Borrelia burgdorferi infection in a natural population of Peromyscus leucopus mice: A longitudinal study in an area where Lyme Borreliosis is highly endemic. J Infect Dis. 2004;189:1515-23. http://dx.doi.org/10.1086/382594

28. Bunikis J, Garpmo U, Tsao J, Berglund J, Fish D, Barbour AG. Sequence typing reveals extensive strain diversity of the Lyme borreliosis agents Borrelia burgdorferi in North America and Borrelia afzelii in Europe. Microbiology. 2004;150:1741-55. http://dx.doi.org/10.1099/mic.0.26944-0

29. Oliveira A, Menezes F, Barbosa Sousa J, Madi RR, Melo CM. Parasitic and fungal infections in synanthropic rodents in an area of urban expansion, Aracaju, Sergipe State, Brazil. Acta Sci Biol Sci. 2014;36:113-20. http://dx.doi.org/10.4025/ actascibiolsci.v36i1.19760

30. Hornok S, Földvári G, Rigó K, Meli ML, Gönczi E, Répási $A$, et al. Synanthropic rodents and their ectoparasites as carriers of a novel haemoplasma and vector-borne, zoonotic pathogens indoors. Parasit Vectors. 2015;8:27. http://dx.doi.org/10.1186/s13071-014-0630-3

31. Hou X, Xu J, Hao Q, Xu G, Geng Z, Zhang L. Prevalence of Borrelia burgdorferi sensu lato in rodents from Jiangxi, southeastern China region. Int J Clin Exp Med. 2014;7:5563-7.

32. Allan B, Keesing F, Ostfeld RS. Effect of forest fragmentation on Lyme disease risk. Conserv Biol. 2003;17:267-72.

33. Manzano R, Díaz V, Pérez R. Garrapatas: características anatómicas, epidemiológicas y ciclo vital. Detalles de la influencia de las garrapatas sobre la producción y sanidad animal. Parasitología Animal. Salamanca: Instituto de Recursos Naturales y Agrobiología de Salamanca; 2012. p. 1-8.

34. Anderson JF, Johnson RC, Magnarelli LA. Seasonal prevalence of Borrelia burgdorferi in natural populations of white-footed mice, Peromyscus leucopus. J Clin Microbiol. 1987;25:1564-6. 
35. Burgess EC, Wachal MD, Cleven TD. Borrelia burgdorferi infection in dairy cows, rodents, and birds from four Wisconsin dairy farms. Vet Microbiol. 1993;35:61-77. http:// dx.doi.org/10.1016/0378-1135(93)90116-O

36. Humair PF, Peter O, Wallich R, Gern L. Strain variation of Lyme disease spirochetes isolated from Ixodes ricinus ticks and rodents collected in two endemic areas in Switzerland. J Med Entomol. 1995;32:433-8. http://dx.doi.org/10.1093/ jmedent/32.4.433

37. Escudero R, Barral M, Pérez A, Vitutia MM, GarcíaPérez AL, Jiménez $\mathrm{S}$, et al. Molecular and pathogenic characterization of Borrelia burgdorferi sensu lato isolates from Spain. J Clin Microbiol. 2000;38:4026-33.

38. Radzijevskaja J, Paulauskas A, Rosef O. Molecular detection and characterization of Borrelia burgdorferi sensu lato in small rodents. Vet Med Zoot. 2011;55:40-6.

39. Smith RP Jr, Muzaffar SB, Lavers J, Lacombe EH, Cahill BK, Lubelczyk CB, et al. Borrelia garinii in seabird ticks (Ixodes uriae), Atlantic Coast, North America. Emerg Infect Dis. 2006;12:1909-12. http://dx.doi.org/10.3201/ eid1212.060448

40. Rudenko N, Golovchenko M, Hönig V, Mallátová N, Krbková L, Mikulášek $\mathrm{P}$, et al. Detection of Borrelia burgdorferi sensu stricto ospC alleles associated with human Lyme borreliosis worldwide in non-human-biting tick Ixodes affinis and rodent hosts in southeastern United States. Appl Environ Microbiol. 2013;1444-53. http://dx.doi. org/10.1128/AEM.02749-12

41. Qiu WG, Bruno JF, McCaig WD, Xu Y, Livey I, Schriefer ME, et al. Wide distribution of a high-virulence Borrelia burgdorferi clone in Europe and North America. Emerg Infect Dis. 2008;14:1097-104. http://dx.doi.org/10.3201/ eid1407.070880
42. Olsen B, Munster VJ, Wallensten A, Waldenstrom J, Osterhaus AD, Fouchier RA. Global patterns of influenza a virus in wild birds. Science. 2006;312:384-8. http://dx.doi. org/10.1126/science.1122438

43. Comstedt P, Jakobsson T, Bergström S. Global ecology and epidemiology of Borrelia garinii spirochetes. Infect Ecol Epidemiol. 2011;1:10. http://dx.doi.org/10.3402/iee. v1i0.9545

44. Scott JD, Durden LA, Anderson JF. Infection prevalence of Borrelia burgdorferi in ticks collected from songbirds in Far-Western Canada. Open J Anim Sci. 2015;5:232-41. http://dx.doi.org/10.4236/ojas.2015.53027

45. Steere AC, Coburn J, Glickstein L. The emergence of Lyme disease. J Clin Invest. 2004;113:1093-101. http://dx. doi.org/dx.doi.org/10.1172/JCl21681

46. Will G, Jauris S, Schwab E, Busch U, Rössler D, SoutschekE, et al. Sequence analysis of OspA genes shows homogeneity within Borrelia burgdorferi sensu stricto and Borrelia afzelii strains but reveals major subgroups within the Borrelia gariniis pecies. Med Microbiol Immunol. 1995;184:73-80.

47. Kurtenbach K, De Michelis S, Sewell HS, Etti S, Schäfer SM, Holmes E, et al. The key roles of selection and migration in the ecology of Lyme borreliosis. Int $\mathrm{J}$ Med Microbiol. 2002;33:152-4. http://dx.doi.org/10.1016/S14384221(02)80029-7

48. Rudenko N, Golovchenco M, Belfiore NM, Grubhoffer L, Oliver JH Jr. Divergence of Borrelia burgdorferi sensu lato spirochetes could be driven by the host: Diversity of Borrelia strains isolated from ticks feeding on a single bird. Parasit Vectors. 2014;7:4. http://dx.doi.org/10.1186/1756-3305-7-4 